MATEMATIKA, 2017, Volume 33, Number 1, 87-95

(C) Penerbit UTM Press. All rights reserved

\title{
Spectrum of commuting graphs of some classes of finite groups
}

\author{
${ }^{1}$ Jutirekha Dutta and ${ }^{2}$ Rajat Kanti Nath \\ ${ }^{1,2}$ Department of Mathematical Sciences, Tezpur University \\ Napaam-784028, Sonitpur, Assam, India \\ e-mail: ${ }^{1}$ jutirekhadutta@yahoo.com, ${ }^{2}$ rajatkantinath@yahoo.com (corresponding author)
}

\begin{abstract}
In this paper, we initiate the study of spectrum of the commuting graphs of finite non-abelian groups. We first compute the spectrum of this graph for several classes of finite groups, in particular AC-groups. We show that the commuting graphs of finite non-abelian AC-groups are integral. We also show that the commuting graph of a finite non-abelian group $G$ is integral if $G$ is not isomorphic to the symmetric group of degree 4 and the commuting graph of $G$ is planar. Further, it is shown that the commuting graph of $G$ is integral if its commuting graph is toroidal.
\end{abstract}

Keywords Commuting graph; Spectrum; Integral Graph; Finite Group.

2010 Mathematics Subject Classification 20D99; 05C50, 15A18, 05C25.

\section{Introduction}

Let $G$ be a finite group with centre $Z(G)$. The commuting graph of a non-abelian group $G$, denoted by $\Gamma_{G}$, is a simple undirected graph whose vertex set is $G \backslash Z(G)$, and two vertices $x$ and $y$ are adjacent if and only if $x y=y x$. Various aspects of commuting graphs of different finite groups can be found in [1-6]. In [7], the authors have studied the Laplacian spectrum of non-commuting graphs of some classes of finite non-abelian groups. In this paper, we initiate the study of spectrum of commuting graphs of finite non-abelian groups. Recall that the spectrum of a graph $\mathcal{G}$ denoted by $\operatorname{Spec}(\mathcal{G})$ is the set $\left\{\lambda_{1}^{k_{1}}, \lambda_{2}^{k_{2}}, \ldots, \lambda_{n}^{k_{n}}\right\}$, where $\lambda_{1}, \lambda_{2}, \ldots, \lambda_{n}$ are the eigenvalues of the adjacency matrix of $\mathcal{G}$ with multiplicities $k_{1}, k_{2}, \ldots, k_{n}$, respectively. A graph $\mathcal{G}$ is called integral if $\operatorname{Spec}(\mathcal{G})$ contains only integers. It is well known that the complete graph $K_{n}$ on $n$ vertices is integral. Moreover, if $\mathcal{G}$ is the disjoint union of some complete graphs then also it is integral. The notion of integral graph was introduced by Harary and Schwenk [8] in the year 1974. A very impressive survey on integral graphs can be found in [9].

We observe that the commuting graph of a non abelian finite AC-group is disjoint union of some complete graphs. Therefore, commuting graphs of such groups are integral. In general it is difficult to classify all finite non-abelian groups whose commuting graphs are integral. As applications of our results together with some other known results, in Section 3 , we show that the commuting graph of a finite non-abelian group $G$ is integral if $G$ is not isomorphic to $S_{4}$, the symmetric group of degree 4, and the commuting graph of $G$ is planar. We also show that the commuting graph of a finite non-abelian group $G$ is integral if the commuting graph of $G$ is toroidal. Recall that the genus of a graph is the smallest non-negative integer $n$ such that the graph can be embedded on the surface obtained by attaching $n$ handles to a sphere. A graph is said to be planar or toroidal if the genus of the graph is zero or one respectively. It is worth mentioning that Afkhami et al. [10] and Das et al. [11] have classified all finite non-abelian groups whose commuting graphs are planar or toroidal recently. 


\section{Computing spectrum}

It is well known that the complete graph $K_{n}$ on $n$ vertices is integral and $\operatorname{Spec}\left(K_{n}\right)$ is given by $\left\{(-1)^{n-1},(n-1)^{1}\right\}$. Further, if $\mathcal{G}=K_{m_{1}} \sqcup K_{m_{2}} \sqcup \cdots \sqcup K_{m_{l}}$, where $K_{m_{i}}$ are complete graphs on $m_{i}$ vertices for $1 \leq i \leq l$, then

$$
\operatorname{Spec}(\mathcal{G})=\left\{(-1)^{\sum_{i=1}^{l} m_{i}-l},\left(m_{1}-1\right)^{1},\left(m_{2}-1\right)^{1}, \ldots,\left(m_{l}-1\right)^{1}\right\} .
$$

If $m_{1}=m_{2}=\cdots=m_{l}=m$ then we write $\mathcal{G}=l K_{m}$ and in that case $\operatorname{Spec}(\mathcal{G})=$ $\left\{(-1)^{l(m-1)},(m-1)^{l}\right\}$.

In this section, we compute the spectrum of the commuting graphs of different families of finite non-abelian AC-groups. A group $G$ is called an AC-group if $C_{G}(x):=\{y \in G: x y=$ $y x\}$ is abelian for all $x \in G \backslash Z(G)$. Various aspects of AC-groups can be found in [11-13]. The following lemma plays an important role in computing spectrum of commuting graphs of AC-groups.

Lemma 2.1 Let $G$ be a finite non-abelian AC-group. Then the commuting graph of $G$ is given by

$$
\Gamma_{G}=\bigsqcup_{i=1}^{n} K_{\left|X_{i}\right|-|Z(G)|}
$$

where $X_{1}, \ldots, X_{n}$ are the distinct centralizers of non-central elements of $G$.

Proof Let $G$ be a finite non-abelian AC-group and $X_{1}, \ldots, X_{n}$ be the distinct centralizers of non-central elements of $G$. Let $X_{i}=C_{G}\left(x_{i}\right)$ where $x_{i} \in G \backslash Z(G)$ and $1 \leq i \leq n$. Let $x, y \in X_{i} \backslash Z(G)$ for some $i$ and $x \neq y$ then, since $G$ an AC-group, there is an edge between $x$ and $y$ in the commuting graph of $G$. Suppose that $x \in\left(X_{i} \cap X_{j}\right) \backslash Z(G)$ for some $1 \leq i \neq j \leq n$. Then $\left[x, x_{i}\right]=1$ and $\left[x, x_{j}\right]=1$. Hence, by Lemma 3.6 of [12] we have $C_{G}(x)=C_{G}\left(x_{i}\right)=C_{G}\left(x_{j}\right)$, a contradiction. Therefore, $X_{i} \cap X_{j}=Z(G)$ for any $1 \leq i \neq j \leq n$. This shows that $\Gamma_{G}=\cup_{i=1}^{n} K_{\left|X_{i}\right|-|Z(G)|}$.

Theorem 2.1 Let $G$ be a finite non-abelian AC-group. Then the spectrum of the commuting graph of $G$ is given by

$$
\left\{(-1)^{\sum_{i=1}^{n}\left|X_{i}\right|-n(|Z(G)|+1)},\left(\left|X_{1}\right|-|Z(G)|-1\right)^{1}, \ldots,\left(\left|X_{n}\right|-|Z(G)|-1\right)^{1}\right\}
$$

where $X_{1}, \ldots, X_{n}$ are the distinct centralizers of non-central elements of $G$.

Proof The proof follows from Lemma 2.1 and (1).

Corollary 2.1 Let $G$ be a finite non-abelian $A C$-group and $A$ be any finite abelian group. Then the spectrum of the commuting graph of $G \times A$ is given by

$$
\begin{aligned}
& \left\{(-1)^{\left.|A|\right|_{i=1} ^{n}\left(\left|X_{i}\right|-n|Z(G)|\right)-n},\left(|A|\left(\left|X_{1}\right|-|Z(G)|\right)-1\right)\right)^{1}, \ldots, \\
& \left.\left.\left(|A|\left(\left|X_{n}\right|-|Z(G)|\right)-1\right)\right)^{1}\right\}
\end{aligned}
$$

where $X_{1}, \ldots, X_{n}$ are the distinct centralizers of non-central elements of $G$. 
Proof It is easy to see that $Z(G \times A)=Z(G) \times A$ and $X_{1} \times A, X_{2} \times A, \ldots, X_{n} \times A$ are the distinct centralizers of non-central elements of $G \times A$. Therefore, if $G$ is an AC-group then $G \times A$ is also an AC-group. Hence, the result follows from Theorem 2.1.

Now we compute the spectrum of the commuting graphs of some particular families of AC-groups. We begin with the well-known family of quasidihedral groups.

Proposition 2.1 The spectrum of the commuting graph of the quasidihedral group $Q D_{2^{n}}=$ $\left\langle a, b: a^{2^{n-1}}=b^{2}=1, b a b^{-1}=a^{2^{n-2}-1}\right\rangle$, where $n \geq 4$, is given by

$$
\operatorname{Spec}\left(\Gamma_{Q D_{2}}\right)=\left\{(-1)^{2^{n}-2^{n-2}-3}, 1^{2^{n-2}},\left(2^{n-1}-3\right)^{1}\right\} .
$$

Proof It is well-known that $Z\left(Q D_{2^{n}}\right)=\left\{1, a^{2^{n-2}}\right\}$. Also

$$
C_{Q D_{2^{n}}}(a)=C_{Q D_{2^{n}}}\left(a^{i}\right)=\langle a\rangle \text { for } 1 \leq i \leq 2^{n-1}-1, i \neq 2^{n-2}
$$

and

$$
C_{Q D_{2^{n}}}\left(a^{j} b\right)=\left\{1, a^{2^{n-2}}, a^{i} b, a^{i+2^{n-2}} b\right\} \text { for } 1 \leq j \leq 2^{n-2}
$$

are the only centralizers of non-central elements of $Q D_{2^{n}}$. Note that these centralizers are abelian subgroups of $Q D_{2^{n}}$. Therefore, by Lemma 2.1

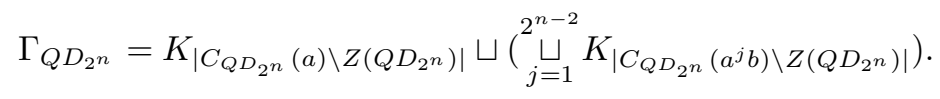

That is, $\Gamma_{Q D_{2^{n}}}=K_{2^{n-1}-2} \sqcup 2^{n-2} K_{2}$, since $\left|C_{Q D_{2^{n}}}(a)\right|=2^{n-1},\left|C_{Q D_{2^{n}}}\left(a^{j} b\right)\right|=4$ for $1 \leq j \leq 2^{n-2}$ and $\left|Z\left(Q D_{2^{n}}\right)\right|=2$. Hence, the result follows from (1).

Proposition 2.2 The spectrum of the commuting graph of the projective special linear group $P S L\left(2,2^{k}\right)$, where $k \geq 2$, is given by

$$
\left\{(-1)^{2^{3 k}-2^{2 k}-2^{k+1}-2},\left(2^{k}-1\right)^{2^{k-1}\left(2^{k}-1\right)},\left(2^{k}-2\right)^{2^{k}+1},\left(2^{k}-3\right)^{2^{k-1}\left(2^{k}+1\right)}\right\} .
$$

Proof We know that $\operatorname{PSL}\left(2,2^{k}\right)$ is a non-abelian group of order $2^{k}\left(2^{2 k}-1\right)$ with trivial center. By Proposition 3.21 of [12], the set of centralizers of non-trivial elements of $\operatorname{PSL}\left(2,2^{k}\right)$ is given by

$$
\left\{x P x^{-1}, x A x^{-1}, x B x^{-1}: x \in P S L\left(2,2^{k}\right)\right\}
$$

where $P$ is an elementary abelian $\quad 2$-subgroup and $A, \quad B$ are cyclic subgroups of $P S L\left(2,2^{k}\right)$ having order $2^{k}, 2^{k}-1$ and $2^{k}+1$ respectively. Also the number of conjugates of $P, A$ and $B$ in $\operatorname{PSL}\left(2,2^{k}\right)$ are $2^{k}+1,2^{k-1}\left(2^{k}+1\right)$ and $2^{k-1}\left(2^{k}-1\right)$ respectively. Note that $P S L\left(2,2^{k}\right)$ is a AC-group and so, by Lemma 2.1, the commuting graph of $P S L\left(2,2^{k}\right)$ is given by

$$
\left(2^{k}+1\right) K_{\left|x P x^{-1}\right|-1} \sqcup 2^{k-1}\left(2^{k}+1\right) K_{\left|x A x^{-1}\right|-1} \sqcup 2^{k-1}\left(2^{k}-1\right) K_{\left|x B x^{-1}\right|-1} .
$$

That is, $\Gamma_{P S L\left(2,2^{k}\right)}=\left(2^{k}+1\right) K_{2^{k}-1} \sqcup 2^{k-1}\left(2^{k}+1\right) K_{2^{k}-2} \sqcup 2^{k-1}\left(2^{k}-1\right) K_{2^{k}}$. Hence, the result follows from (1).

Proposition 2.3 The spectrum of the commuting graph of the general linear group $G L(2, q)$, where $q=p^{n}>2$ and $p$ is a prime integer, is given by

$$
\left\{(-1)^{q^{4}-q^{3}-2 q^{2}-q},\left(q^{2}-3 q+1\right)^{q(q+1) / 2},\left(q^{2}-q-1\right)^{q(q-1) / 2},\left(q^{2}-2 q\right)^{q+1}\right\} .
$$


Proof We have $|G L(2, q)|=\left(q^{2}-1\right)\left(q^{2}-q\right)$ and $|Z(G L(2, q))|=q-1$. By Proposition 3.26 of [12], the set of centralizers of non-central elements of $G L(2, q)$ is given by

$$
\left\{x D x^{-1}, x I x^{-1}, x P Z(G L(2, q)) x^{-1}: x \in G L(2, q)\right\}
$$

where $D$ is the subgroup of $G L(2, q)$ consisting of all diagonal matrices, $I$ is a cyclic subgroup of $G L(2, q)$ having order $q^{2}-1$ and $P$ is the Sylow $p$-subgroup of $G L(2, q)$ consisting of all upper triangular matrices with 1 in the diagonal. The orders of $D$ and $P Z(G L(2, q))$ are $(q-1)^{2}$ and $q(q-1)$ respectively. Also the number of conjugates of $D, I$ and $P Z(G L(2, q))$ in $G L(2, q)$ are $q(q+1) / 2, q(q-1) / 2$ and $q+1$ respectively. Since $G L(2, q)$ is an AC-group (see Lemma 3.5 of $[12]$ ), by Lemma 2.1 we have $\Gamma_{G L(2, q)}=$

$$
\frac{q(q+1)}{2} K_{\left|x D x^{-1}\right|-q+1} \sqcup \frac{q(q-1)}{2} K_{\left|x I x^{-1}\right|-q+1} \sqcup(q+1) K_{\left|x P Z(G L(2, q)) x^{-1}\right|-q+1} \cdot
$$

That is, $\Gamma_{G L(2, q)}=\frac{q(q+1)}{2} K_{q^{2}-3 q+2} \sqcup \frac{q(q-1)}{2} K_{q^{2}-q} \sqcup(q+1) K_{q^{2}-2 q+1}$. Hence, the result follows from (1).

Theorem 2.2 Let $G$ be a finite group and $\frac{G}{Z(G)} \cong S z(2)$, where $S z(2)$ is the Suzuki group presented by $\left\langle a, b: a^{5}=b^{4}=1, b^{-1} a b=a^{2}\right\rangle$. Then

$$
\operatorname{Spec}\left(\Gamma_{G}\right)=\left\{(-1)^{19|Z(G)|-6},(4|Z(G)|-1)^{1},(3|Z(G)|-1)^{5}\right\} .
$$

Proof We have

$$
\frac{G}{Z(G)}=\left\langle a Z(G), b Z(G): a^{5} Z(G)=b^{4} Z(G)=Z(G), b^{-1} a b Z(G)=a^{2} Z(G)\right\rangle .
$$

Observe that

$$
\begin{array}{ll}
C_{G}(a) & =Z(G) \sqcup a Z(G) \sqcup a^{2} Z(G) \sqcup a^{3} Z(G) \sqcup a^{4} Z(G), \\
C_{G}(a b) & =Z(G) \sqcup a b Z(G) \sqcup a^{4} b^{2} Z(G) \sqcup a^{3} b^{3} Z(G), \\
C_{G}\left(a^{2} b\right) & =Z(G) \sqcup a^{2} b Z(G) \sqcup a^{3} b^{2} Z(G) \sqcup a b^{3} Z(G), \\
C_{G}\left(a^{2} b^{3}\right) & =Z(G) \sqcup a^{2} b^{3} Z(G) \sqcup a b^{2} Z(G) \sqcup a^{4} b Z(G), \\
C_{G}(b) & =Z(G) \sqcup b Z(G) \sqcup b^{2} Z(G) \sqcup b^{3} Z(G) \quad \text { and } \\
C_{G}\left(a^{3} b\right) & =Z(G) \sqcup a^{3} b Z(G) \sqcup a^{2} b^{2} Z(G) \sqcup a^{4} b^{3} Z(G)
\end{array}
$$

are the only centralizers of non-central elements of $G$. Also note that these centralizers are abelian subgroups of $G$. Thus $G$ is an AC-group. By Lemma 2.1, we have

$$
\Gamma_{G}=K_{4|Z(G)|} \sqcup 5 K_{3|Z(G)|}
$$

since $\left|C_{G}(a)\right|=5|Z(G)|$ and

$$
\left|C_{G}(a b)\right|=\left|C_{G}\left(a^{2} b\right)\right|=\left|C_{G}\left(a^{2} b^{3}\right)\right|=\left|C_{G}(b)\right|=\left|C_{G}\left(a^{3} b\right)\right|=4|Z(G)| .
$$

Therefore, by (1), the result follows. 
Proposition 2.4 Let $F=G F\left(2^{n}\right), n \geq 2$ and $\vartheta$ be the Frobenius automorphism of $F$, $i$. e., $\vartheta(x)=x^{2}$ for all $x \in F$. Then the spectrum of the commuting graph of the group

$$
A(n, \vartheta)=\left\{U(a, b)=\left[\begin{array}{ccc}
1 & 0 & 0 \\
a & 1 & 0 \\
b & \vartheta(a) & 1
\end{array}\right]: a, b \in F\right\} .
$$

under matrix multiplication given by $U(a, b) U\left(a^{\prime}, b^{\prime}\right)=U\left(a+a^{\prime}, b+b^{\prime}+a^{\prime} \vartheta(a)\right)$ is

$$
\operatorname{Spec}\left(\Gamma_{A(n, \vartheta)}\right)=\left\{(-1)^{\left(2^{n}-1\right)^{2}},\left(2^{n}-1\right)^{2^{n}-1}\right\} .
$$

Proof Note that $Z(A(n, \vartheta))=\{U(0, b): b \in F\}$ and so $|Z(A(n, \vartheta))|=2^{n}-1$. Let $U(a, b)$ be a non-central element of $A(n, \vartheta)$. It can be seen that the centralizer of $U(a, b)$ in $A(n, \vartheta)$ is $Z(A(n, \vartheta)) \sqcup U(a, 0) Z(A(n, \vartheta))$. Clearly $A(n, \vartheta)$ is an AC-group and so by Lemma 2.1 we have $\Gamma_{A(n, \vartheta)}=\left(2^{n}-1\right) K_{2^{n}}$. Hence the result follows by $(1)$.

Proposition 2.5 Let $F=G F\left(p^{n}\right), p$ be a prime. Then the spectrum of the commuting graph of the group

$$
A(n, p)=\left\{V(a, b, c)=\left[\begin{array}{lll}
1 & 0 & 0 \\
a & 1 & 0 \\
b & c & 1
\end{array}\right]: a, b, c \in F\right\} .
$$

under matrix multiplication $V(a, b, c) V\left(a^{\prime}, b^{\prime}, c^{\prime}\right)=V\left(a+a^{\prime}, b+b^{\prime}+c a^{\prime}, c+c^{\prime}\right)$ is

$$
\operatorname{Spec}\left(\Gamma_{A(n, p)}\right)=\left\{(-1)^{p^{3 n}-2 p^{n}-1},\left(p^{2 n}-p^{n}-1\right)^{p^{n}+1}\right\} .
$$

Proof We have $Z(A(n, p))=\{V(0, b, 0): b \in F\}$ and so $|Z(A(n, p))|=p^{n}$. The centralizers of non-central elements of $A(n, p)$ are given by

(i) If $b, c \in F$ and $c \neq 0$ then the centralizer of $V(0, b, c)$ in $A(n, p)$ is $\left\{V\left(0, b^{\prime}, c^{\prime}\right): b^{\prime}, c^{\prime} \in F\right\}$ having order $\left|p^{2 n}\right|$.

(ii) If $a, b \in F$ and $a \neq 0$ then the centralizer of $V(a, b, 0)$ in $A(n, p)$ is $\left\{V\left(a^{\prime}, b^{\prime}, 0\right): a^{\prime}, b^{\prime} \in F\right\}$ having order $\left|p^{2 n}\right|$.

(iii) If $a, b, c \in F$ and $a \neq 0, c \neq 0$ then the centralizer of $V(a, b, c)$ in $A(n, p)$ is $\left\{V\left(a^{\prime}, b^{\prime}, c a^{\prime} a^{-1}\right)\right.$ : $\left.a^{\prime}, b^{\prime} \in F\right\}$ having order $\left|p^{2 n}\right|$.

It can be seen that all the centralizers of non-central elements of $A(n, p)$ are abelian. Hence $A(n, p)$ is an AC-group and so

$$
\Gamma_{A(n, p)}=K_{p^{2 n}-p^{n}} \sqcup K_{p^{2 n}-p^{n}} \sqcup\left(p^{n}-1\right) K_{p^{2 n}-p^{n}}=\left(p^{n}+1\right) K_{p^{2 n}-p^{n}} .
$$

Hence the result follows from (1).

We would like to mention here that the groups considered in Proposition 2.4-2.5 are constructed by Hanaki (see [14]). These groups are also considered in [15], in order to compute their numbers of distinct centralizers. 


\section{Some applications}

In this section, we show that the commuting graph of a finite non-abelian group $G$ is integral if $G$ is not isomorphic to $S_{4}$ and the commuting graph of $G$ is planar. We also show that the commuting graph of a finite non-abelian group $G$ is integral if the commuting graph of $G$ is toroidal. We shall use the following results.

Theorem 3.1 Let $G$ be a finite group such that $\frac{G}{Z(G)} \cong \mathbb{Z}_{2} \times \mathbb{Z}_{2}$. Then

$$
\operatorname{Spec}\left(\Gamma_{G}\right)=\left\{(-1)^{3|Z(G)|-3},(|Z(G)|-1)^{3}\right\} .
$$

Proof The result follows from Theorem 2.1 noting that $G$ is an AC-group with 3 distinct centralizers of non-central elements and all of them have order $2|Z(G)|$.

Proposition 3.1 Let $D_{2 m}=\left\langle a, b: a^{m}=b^{2}=1, b a b^{-1}=a^{-1}\right\rangle$ be the dihedral group of order $2 m$, where $m>2$. Then

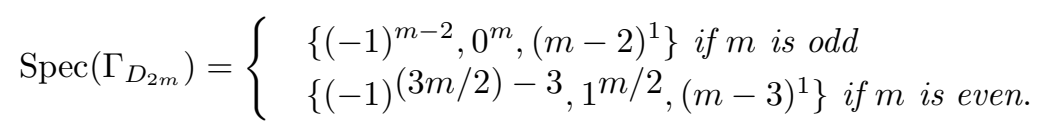

Proof Note that $D_{2 m}$ is a non-abelian AC-group. If $m$ is even then $\left|Z\left(D_{2 m}\right)\right|=2$ and $D_{2 m}$ has $\frac{m}{2}+1$ distinct centralizers of non-central elements. Out of these centralizers one has order $m$ and the rests have order 4 . Therefore $\Gamma_{D_{2 m}}=K_{m-2} \sqcup \frac{m}{2} K_{2}$. If $m$ is odd then $\left|Z\left(D_{2 m}\right)\right|=1$ and $D_{2 m}$ has $m+1$ distinct centralizers of non-central elements. In this case, one centralizer has order $m$ and the rests have order 2. Therefore $\Gamma_{D_{2 m}}=K_{m-1} \sqcup m K_{1}$. Hence the result follows from (1).

Proposition 3.2 The spectrum of the commuting graph of the generalized quaternion group $Q_{4 n}=\left\langle x, y: y^{2 n}=1, x^{2}=y^{n}, x y x^{-1}=y^{-1}\right\rangle$, where $n \geq 2$, is given by

$$
\operatorname{Spec}\left(\Gamma_{Q_{4 n}}\right)=\left\{(-1)^{3 n-3}, 1^{n},(2 n-3)^{1}\right\} .
$$

Proof Note that $Q_{4 n}$ is a non-abelian AC-group with $n+1$ distinct centralizers of noncentral elements. Out of these centralizers one has order $2 n$ and the rests have order 4 . Also $\left|Z\left(Q_{4 n}\right)\right|=2$. Therefore $\Gamma_{Q_{4 n}}=K_{2 n-2} \sqcup n K_{2}$. Hence the result follows from (1).

As an application of Theorem 3.1 we have the following lemma.

Lemma 3.1 Let $G$ be a group isomorphic to any of the following groups

(i) $\mathbb{Z}_{2} \times D_{8}$

(ii) $\mathbb{Z}_{2} \times Q_{8}$

(iii) $M_{16}=\left\langle a, b: a^{8}=b^{2}=1, b a b=a^{5}\right\rangle$

(iv) $\mathbb{Z}_{4} \rtimes \mathbb{Z}_{4}=\left\langle a, b: a^{4}=b^{4}=1, b a b^{-1}=a^{-1}\right\rangle$

(v) $D_{8} * \mathbb{Z}_{4}=\left\langle a, b, c: a^{4}=b^{2}=c^{2}=1, a b=b a, a c=c a, b c=a^{2} c b\right\rangle$

(vi) $S G(16,3)=\left\langle a, b: a^{4}=b^{4}=1, a b=b^{-1} a^{-1}, a b^{-1}=b a^{-1}\right\rangle$.

Then $\operatorname{Spec}\left(\Gamma_{G}\right)=\left\{(-1)^{9}, 3^{3}\right\}$. 
Proof If $G$ is isomorphic to any of the above listed groups, then $|G|=16$ and $|Z(G)|=4$. Therefore, $\frac{G}{Z(G)} \cong \mathbb{Z}_{2} \times \mathbb{Z}_{2}$. Thus the result follows from Theorem 3.1.

The next lemma is also useful in this section.

Lemma 3.2 Let $G$ be a non-abelian group of order $p q$, where $p$ and $q$ are primes with $p \mid(q-1)$. Then

$$
\operatorname{Spec}\left(\Gamma_{G}\right)=\left\{(-1)^{p q-q-1},(p-2)^{q},(q-2)^{1}\right\}
$$

Proof It is easy to see that $|Z(G)|=1$ and $G$ is an AC-group. Also the centralizers of non-central elements of $G$ are precisely the Sylow subgroups of $G$. The number of Sylow $q$-subgroups and Sylow $p$-subgroups of $G$ are one and $q$ respectively. Therefore, by Lemma 2.1 we have $\Gamma_{G}=K_{q-1} \sqcup q K_{p-1}$. Hence, the result follows from (1).

Now we state and proof the main results of this section.

Theorem 3.2 Let $\Gamma_{G}$ be the commuting graph of a finite non-abelian group $G$. If $G$ is not isomorphic to $S_{4}$ and $\Gamma_{G}$ is planar then $\Gamma_{G}$ is integral.

Proof By Theorem 2.2 of [10] we have that $\Gamma_{G}$ is planar if and only if $G$ is isomorphic to either $D_{6}, D_{8}, D_{10}, D_{12}, Q_{8}, Q_{12}, \mathbb{Z}_{2} \times D_{8}, \mathbb{Z}_{2} \times Q_{8}, M_{16}, \mathbb{Z}_{4} \rtimes \mathbb{Z}_{4}, D_{8} * \mathbb{Z}_{4}, S G(16,3), A_{4}$, $A_{5}, S_{4}, S L(2,3)$ or $S z(2)=\left\langle a, b: a^{5}=b^{4}=1, b^{-1} a b=a^{3}\right\rangle$.

If $G \cong D_{6}, D_{8}, D_{10}$ or $D_{12}$ then by Proposition 3.1 , one may conclude that $\Gamma_{G}$ is integral. If $G \cong Q_{8}$ or $Q_{12}$ then by Proposition $3.2, \Gamma_{G}$ becomes integral. If $G \cong \mathbb{Z}_{2} \times D_{8}, \mathbb{Z}_{2} \times$ $Q_{8}, M_{16}, \mathbb{Z}_{4} \rtimes \mathbb{Z}_{4}, D_{8} * \mathbb{Z}_{4}$ or $S G(16,3)$ then by Lemma $3.1, \Gamma_{G}$ becomes integral.

If $G \cong A_{4}=\left\langle a, b: a^{2}=b^{3}=(a b)^{3}=1\right\rangle$ then the distinct centralizers of non-central elements of $G$ are $C_{G}(a)=\left\{1, a, b a b^{2}, b^{2} a b\right\}, C_{G}(b)=\left\{1, b, b^{2}\right\}, C_{G}(a b)=\left\{1, a b, b^{2} a\right\}, C_{G}(b a)=$ $\left\{1, b a, a b^{2}\right\}$ and $C_{G}(a b a)=\{1, a b a, b a b\}$. Note that these centralizers are abelian subgroups of $G$. Therefore, $\Gamma_{G}=K_{3} \sqcup 4 K_{2}$ and

$$
\operatorname{Spec}\left(\Gamma_{G}\right)=\left\{(-1)^{6}, 2^{1}, 1^{4}\right\} .
$$

Thus $\Gamma_{G}$ is integral.

If $G \cong S z(2)$ then by Theorem 2.2 , we have

$$
\operatorname{Spec}\left(\Gamma_{G}\right)=\left\{(-1)^{13},(3)^{1},(2)^{5}\right\} .
$$

Hence, $\Gamma_{G}$ is integral.

If $G$ is isomorphic to

$$
\begin{aligned}
& S L(2,3)=\left\langle a, b, c: a^{3}=b^{4}=1, b^{2}=c^{2}\right. \\
& \left.\quad c^{-1} b c=b^{-1}, a^{-1} b a=b^{-1} c^{-1}, a^{-1} c a=b^{-1}\right\rangle
\end{aligned}
$$

then $Z(G)=\left\{1, b^{2}\right\}$. It can be seen that

$$
\begin{array}{ll}
C_{G}(b) & =\left\{1, b, b^{2}, b^{3}\right\}=\langle b\rangle, \\
C_{G}(c) & =\left\{1, c, c^{2}, c^{3}\right\}=\langle c\rangle, \\
C_{G}(b c) & =\left\{1, b^{2}, b c, c b\right\}=\langle b c\rangle, \\
C_{G}\left(a^{2} b^{2}\right) & =\left\{1, b^{2}, a, a^{2}, a^{2} b^{2}, a b^{2}\right\}=\left\langle a^{2} b^{2}\right\rangle, \\
C_{G}(a c) & =\left\{1, b^{2}, a c, c a^{2}, a^{2} b c, a b^{2} c\right\}=\langle a c\rangle, \\
C_{G}(c a) & =\left\{1, b^{2}, c a, a^{2} c, b a^{2}, a b\right\}=\langle c a\rangle \text { and } \\
C_{G}\left(a^{2} b\right) & =\left\{1, b^{2}, a^{2} b, b a, b^{3} a,(b a)^{2}\right\}=\left\langle a^{2} b\right\rangle
\end{array}
$$


are the only distinct centralizers of non-central elements of $G$. Note that these centralizers are abelian subgroups of $G$. Therefore, $\Gamma_{G}=3 K_{2} \sqcup 4 K_{4}$ and

$$
\operatorname{Spec}\left(\Gamma_{G}\right)=\left\{(-1)^{15}, 1^{3}, 3^{4}\right\} .
$$

Thus $\Gamma_{G}$ is integral.

If $G \cong A_{5}$ then by Proposition 2.2 , we have

$$
\operatorname{Spec}\left(\Gamma_{G}\right)=\left\{(-1)^{38}, 1^{10}, 2^{5}, 3^{6}\right\}
$$

noting that $P S L(2,4) \cong A_{5}$. Thus $\Gamma_{G}$ is integral.

Finally, if $G \cong S_{4}$ then it can be seen that the characteristic polynomial of $\Gamma_{G}$ is $(x-1)^{7}(x+1)^{10}\left(x^{2}-5\right)^{2}\left(x^{2}-3 x-2\right)$ and so

$$
\operatorname{Spec}\left(\Gamma_{G}\right)=\left\{1^{7},(-1)^{10},(\sqrt{5})^{2},(-\sqrt{5})^{2},\left(\frac{3+\sqrt{17}}{2}\right)^{1},\left(\frac{3-\sqrt{17}}{2}\right)^{1}\right\} .
$$

Hence, $\Gamma_{G}$ is not integral. This completes the proof.

In [10, Theorem 2.3], Afkhami et al. have classified all finite non-abelian groups whose commuting graphs are toroidal. Unfortunately, the statement of Theorem 2.3 in [10] is printed incorrectly. We list the correct version of [10, Theorem 2.3] below, since we are going to use this.

Theorem 3.3 Let $G$ be a finite non-abelian group. Then $\Gamma_{G}$ is toroidal if and only if $\Gamma_{G}$ is projective if and only if $G$ is isomorphic to either $D_{14}, D_{16}, Q_{16}, Q D_{16}, D_{6} \times \mathbb{Z}_{3}, A_{4} \times \mathbb{Z}_{2}$ or $\mathbb{Z}_{7} \rtimes \mathbb{Z}_{3}$.

Theorem 3.4 Let $\Gamma_{G}$ be the commuting graph of a finite non-abelian group $G$. Then $\Gamma_{G}$ is integral if $\Gamma_{G}$ is toroidal.

Proof By Theorem 3.3 we have that $\Gamma_{G}$ is toroidal if and only if $G$ is isomorphic to either $D_{14}, D_{16}, Q_{16}, Q D_{16}, D_{6} \times \mathbb{Z}_{3}, A_{4} \times \mathbb{Z}_{2}$ or $\mathbb{Z}_{7} \rtimes \mathbb{Z}_{3}$.

If $G \cong D_{14}$ or $D_{16}$ then by Proposition 3.1 , one may conclude that $\Gamma_{G}$ is integral. If $G \cong Q_{16}$ then by Proposition $3.2, \Gamma_{G}$ becomes integral. If $G \cong Q D_{16}$ then by Proposition 2.1, $\Gamma_{G}$ becomes integral. If $G \cong \mathbb{Z}_{7} \rtimes \mathbb{Z}_{3}$ then $\Gamma_{G}$ is integral, by Lemma 3.2. If $G$ is isomorphic to $D_{6} \times \mathbb{Z}_{3}$ or $A_{4} \times \mathbb{Z}_{2}$ then $\Gamma_{G}$ becomes integral by Corollary 2.1, since $D_{6}$ and $A_{4}$ are AC-groups. This completes the proof.

We shall conclude the paper with the following result.

Proposition 3.3 Let $\Gamma_{G}$ be the commuting graph of a finite non-abelian group $G$. Then $\Gamma_{G}$ is integral if the complement of $\Gamma_{G}$ is planar.

Proof If the complement of $\Gamma_{G}$ is planar then by Proposition 2.3 of [12] we have that $G$ is isomorphic to either $D_{6}, D_{8}$ or $Q_{8}$. If $G \cong D_{6}$ or $D_{8}$ then by Proposition 3.1, $\Gamma_{G}$ is integral. If $G \cong Q_{8}$ then by Proposition $3.2, \Gamma_{G}$ becomes integral. This completes the proof. 


\section{References}

[1] Akbari, S. Mohammadian, A. Radjavi, H. and Raja, P. On the diameters of commuting graphs. Linear Algebra Appl. 2006. 418: 161-176.

[2] Bates, C., Bundy, D., Hart, S. and Rowley, P. A note on commuting graphs for symmetric groups. Electron. J. Combin. 2009. 16: 1-13.

[3] Iranmanesh, A. and Jafarzadeh, A. Characterization of finite groups by their commuting graph. Acta Mathematica Academiae Paedagogicae Nyiregyhaziensis. 2007. 23(1): $7-13$.

[4] Iranmanesh, A. and Jafarzadeh, A. On the commuting graph associated with the symmetric and alternating groups. J. Algebra Appl. 2008. 7(1): 129-146.

[5] Morgan, G. L. and Parker, C. W. The diameter of the commuting graph of a finite group with trivial center. J. Algebra. 2013. 393(1): 41-59.

[6] Parker, C. The commuting graph of a soluble group. Bull. London Math. Soc. 2013. 45(4): 839-848.

[7] Dutta, J. and Nath, R. K. Laplacian spectrum of non-commuting graphs of some finite groups. preprint.

[8] Harary, F. and Schwenk, A. J. Which graphs have integral spectra? Graphs and Combin., Lect. Notes Math. Springer-Verlag, Berlin. 1974. 406: 45-51.

[9] Balińska, K., Cvetković, D., Radosavljević, Z., Simić, S. and Stevanović, D. A survey on integral graphs. Univ. Beograd. Publ. Elektrotehn. Fak. Ser. Mat. 2003. 13: 42-65.

[10] M. Afkhami, Farrokhi, D. G. M. and Khashyarmanesh, K. Planar, toroidal, and projective commuting and non-commuting graphs. Comm. Algebra. 2015. 43(7): 2964-2970.

[11] Das, A. K. and Nongsiang, D. On the genus of the commuting graphs of finite nonabelian groups. Int. Electron. J. Algebra. 2016. 19: 91-109.

[12] Abdollahi, A., Akbari, S. and Maimani, H. R. Non-commuting graph of a group. $J$. Algebra. 2006. 298: 468-492.

[13] Rocke, D. M. p-groups with abelian centralizers. Proc. London Math. Soc. 1975. 30(3): $55-75$.

[14] Hanaki, A. A condition of lengths of conjugacy classes and character degree. Osaka J. Math. 1996. 33: 207-216.

[15] Ashrafi, A. R. On finite groups with a given number of centralizers. Algebra Colloq. 2000. $7(2):$ 139-146. 